\title{
The combined effects of omega-3 polyunsaturated fatty acids and B vitamins on cognition in the older adult: a systematic review
}

\author{
P. Fairbairn ${ }^{1,2}$ and F. Tsofliou ${ }^{1}$ \\ ${ }^{1}$ Faculty of Health and Social Sciences, Bournemouth University, UK and \\ ${ }^{2}$ The School of Health Sciences, Queen Margaret University, Musselburgh, UK
}

Cognition is critical for functional independence in older adults ${ }^{(1)}$. There is now evidence to suggest that there may be an interaction between B vitamins and omega-3 polyunsaturated fatty acids (PUFA), with suggestions that optimising intake of both nutrients may be key to eliciting beneficial effects on cognition ${ }^{(2)}$. A lack of consideration for this interaction could have contributed to variance in results from single nutrient supplementation trials. It is therefore important to examine the current available literature that has investigated the combined effects of omega-3 PUFA and B vitamins. The aim of this systematic review was to determine whether supplementation with a combination of omega-3 PUFAs and B vitamins can prevent cognitive decline in older adults.

The following databases were searched for articles published between January 2010 and December 2020: the Cochrane Library, EMBASE, CINAHL, Scopus, and MEDLINE. Risk of bias was assessed using the Revised Cochrane risk-of-bias tool for randomised trials. Articles were included if they met the following criteria: (1) the study type was a randomised control trial; (2) The mean age of the participants in the study was 60 years or greater; (3) the study intervention provided a combination of omega-3 PUFAs and B vitamins alone or in combination with other nutrients; (4) the study tested for interactions between omega-3 PUFAs and B vitamins by providing omega-3 PUFA alone and also measuring B vitamin status, provided B vitamin supplementation alone and measured omega-3 PUFA status; (4) assessed cognitive function.

The databases identified 4,715 results. A total of 14 papers were included ( $n=4913$; age: $60-70$ y; follow up 24 weeks to 4 years). Eleven studies were RCTs analysing the effects of a combination of omega-3 PUFAs and B vitamins. Nine studies provided multinutrient formulas that included additional active ingredients with two providing only a combination of B vitamins and omega-3 PUFAs with no additional active ingredients. Three included papers were post-hoc analyses of RCTs, one was a study that provided omega-3 PUFA supplements and proceeded to measure homocysteine, with two providing B vitamin supplements and proceeding to measure omega-3 PUFA status. There was heterogeneity in measurement techniques used to assess cognitive function, the cognitive domains that were assessed and the cognitive status of participants at baseline. Cognitive domains tested included global cognition $(n=11)$, executive function $(n=10)$, episodic memory $(n=7)$, processing speed $(n=3)$, semantic memory $(n=3)$, spatial working memory $(n=2)$, attention and concentration $(n=2)$, interference control $(n=1)$ and mental fatigue $(n=1)$.

This review is the first to systematically examine the combination of omega-3 PUFAs and $\mathrm{B}$ vitamins on cognition in older adults. More experimental work providing a combination of omega-3 PUFAs and B vitamins is warranted to better explore how nutrition can impact cognition in the older adult.

\section{References}

1. Murman DL. (2015) Semin Hear 36(3), 111-21.

2. Oulhaj A, Jernerén F, Refsum H, et al. (2016) J Alzheimers Dis 50(2), 547-57. 